\title{
MODELAGEM CINÉTICA DA DESIDRATAÇÃO DE ISOPROPANOL SOBRE ZEÓLITA BETA
}

\author{
H. ENZWEILER ${ }^{1}$, E. B. COUTINHO ${ }^{2}$ e M. SCHWAAB ${ }^{3}$ \\ ${ }^{1}$ Universidade Estadual de Maringá, Departamento de Engenharia Química \\ ${ }^{2}$ Universidade Federal do Rio Grande do Sul, Departamento de Físico-Química \\ ${ }^{3}$ Universidade Federal Santa Maria, Departamento de Engenharia Química \\ E-mail para contato: marcioschwaab@gmail.com
}

\begin{abstract}
RESUMO - A desidratação de isopropanol, muito utilizada como reação modelo para a determinação da acidez de sítios ativos, pode ser empregada para a síntese de propeno a partir de uma fonte renovável. A forma ácida da zeólita Beta, obtida através de síntese hidrotérmica, seguida de troca iônica, foi utilizada como catalisador. A zeólita foi caracterizada por difração de raios-X e fisissorção de $\mathrm{N}_{2}$. A reação foi estudada variandose composição e vazão da alimentação. Nos testes reacionais, propeno foi formado preferencialmente, com apenas pequenas concentrações de éter di-isopropílico a baixas temperaturas, sendo obtidas conversões completas do álcool em algumas condições. Dois modelos cinéticos, baseados em mecanismos do tipo Langmuir-Hinshelwood, foram propostos e ajustados aos dados experimentais, sendo seus parâmetros estimados. Foi obtido um modelo cinético simplificado, após a eliminação de parâmetros não significativos, capaz de representar muito bem a composição dos produtos de reação.
\end{abstract}

\section{INTRODUÇÃO}

Alcoóis, especialmente isopropanol, são largamente utilizados como reagentes de reação modelo para a determinação de propriedades de catalisadores heterogêneos (Bedia et al., 2010). A conversão do isopropanol pode ocorrer por duas rotas, dependendo das características dos sítios ativos do sólido. Em sítios ácidos ocorre a desidratação do álcool, formando propeno e éter di-isopropílico. Já em sítios básicos/metálicos ocorre a desidrogenação do isopropanol. Enquanto a seletividade para a reação de desidratação em relação à desidrogenação indica a característica acido-básica do catalisador, a razão entre os produtos propeno e éter está relaciona à força dos sítios ácidos (Trejo et al., 2012). Dentre os catalisadores ácidos existentes, as zeólitas são amplamente utilizadas, devido a suas propriedades ácidas e sua seletividade de forma. Zeólitas são aluminosilicatos cristalinos, com estrutura tridimensional formada por tetraedros de $\mathrm{SiO}_{4} \mathrm{e}$ $\mathrm{AlO}_{4}$, ligados pelos átomos de oxigênio. Dentre as zeólitas, a zeólita Beta apresenta acidez de moderada a forte, seletividade para olefinas e estabilidade catalítica, propriedades desejadas na reação de desidratação de isopropanol. O objetivo principal deste trabalho é a modelagem cinética da reação de desidratação de isopropanol sobre zeólita Beta. Para tanto, o catalisador foi sintetizado e caracterizado. Os testes catalíticos foram realizados em diferentes condições 


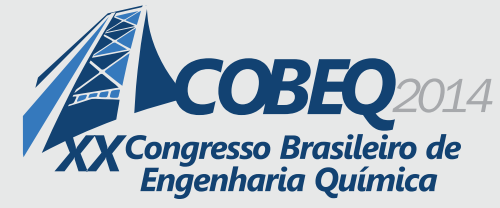

experimentais. Os modelos cinéticos propostos foram ajustados aos dados experimentais.

\section{MATERIAIS E MÉTODOS}

\subsection{Síntese e caracterização da zeólita Beta}

A zeólita Beta foi sintetizada como descrito em Enzweiler et al. (2013), utilizando sílica extraída de cinzas de casca de arroz, pseudoboemita e hidróxido de tetraetilamônio como fontes de silício e alumínio e direcionador de estrutura. A estrutura cristalina do catalisador foi identificada por difração de raios-X, em um difratômetro de raios-X Rigaku modelo Miniflex, com radiação $\mathrm{Cu} \mathrm{K \alpha}$ e fonte de energia com $30 \mathrm{kV}$ e $10 \mathrm{~mA}$. A adsorção/dessorção estática de $\mathrm{N}_{2}$ a $77 \mathrm{~K}$ foi utilizada para análise das propriedades texturais em equipamento ASAP 2020 (Micromeritics).

\subsection{Testes catalíticos de desidratação de isopropanol}

Os testes catalíticos eram realizados em unidade de reação em leito fixo com fluxo contínuo. A unidade era constituída de forno, com controle digital de temperatura e reator de vidro em "U". Os reagentes e produtos da reação eram analisados por cromatografia gasosa, Shimadzu (GC-17A) equipado com coluna capilar HP-PLOT-Q (Agilent Technologies) e com detector tipo ionização em chama (FID). O isopropanol (Synth, pureza $\geq 99,5 \%$ ) era alimentado através do borbulhamento de $\mathrm{N}_{2}$ em um saturador, contendo o reagente, mantido a temperatura controlada em banho termostático.

Nos experimentos de desidratação de isopropanol a forma ácida da zeólita Beta foi utilizada como catalisador. Para garantir que o catalisador estava seco no início da reação, a zeólita era submetida a um tratamento in situ a $200{ }^{\circ} \mathrm{C}$, sob fluxo de $\mathrm{N}_{2}$. As condições reacionais foram variadas de acordo com um planejamento experimental fatorial completo a dois níveis com triplicata no ponto central. Os experimentos eram realizados em seis temperaturas de reação $\left(130,150,160,170,180\right.$ e $\left.200{ }^{\circ} \mathrm{C}\right)$. As variáveis operacionais foram fixadas de acordo com o planejamento experimental apresentado na Tabela 1.

Tabela 1 - Planejamento experimental para desidratação de isopropanol

\begin{tabular}{ccc|ccc}
\hline Experimento & $\begin{array}{c}\text { Temperatura do } \\
\text { saturador }\left({ }^{\circ} \mathrm{C}\right)\end{array}$ & $\begin{array}{c}\text { Vazão de } N_{2} \\
(\mathrm{~mL} / \mathrm{min})\end{array}$ & Experimento & $\begin{array}{c}\text { Temperatura do } \\
\text { saturador }\left({ }^{\circ} \mathrm{C}\right)\end{array}$ & $\begin{array}{c}\text { Vazão de } \mathrm{N}_{2} \\
(\mathrm{~mL} / \mathrm{min})\end{array}$ \\
\hline E1 & $10(-1)$ & $35(-1)$ & E5 & $15(0)$ & $55(0)$ \\
E2 & $10(-1)$ & $75(1)$ & E6 & $15(0)$ & $55(0)$ \\
E3 & $20(-1)$ & $35(-1)$ & E7 & $15(0)$ & $55(0)$ \\
\cline { 4 - 6 } E4 & $20(-1)$ & $75(1)$ & &
\end{tabular}

(Valor normalizado das variáveis)

\subsection{Modelos cinéticos}

A desidratação de isopropanol envolve duas reações com produtos distintos: olefina e éter. 


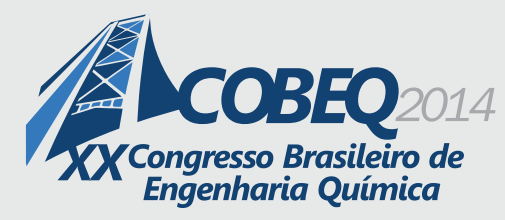

As velocidades de reação de formação de propeno $\left(r_{\text {prope }}\right)$ e de éter di-isopropílico $\left(r_{\text {eter }}\right)$ foram definidas de acordo com os mecanismos de reação. Inicialmente considerou-se que o sistema, apesar da presença do catalisador heterogêneo, seguia o comportamento de reações homogêneas. O modelo resultante é chamado de pseudo-homogêneo. Outros modelos foram desenvolvidos a partir dos mecanismos de reações heterogêneas, através do método de Langmuir-HinshelwoodHougen-Watson (Figueiredo e Ribeiro, 1989).

O mecanismo da desidratação de isopropanol formando propeno foi descrito por três etapas elementares, assim como o mecanismo de formação de éter di-isopropílico. As etapas são a adsorção de isopropanol no sítio ácido (*) da zeólita Beta, R1, seguida das etapas de reação superficial que levam à formação de propeno (R2.1) ou de éter (R2.2); e finalmente a dessorção dos produtos, propeno (R3.1) e éter (R3.2). Foram consideradas duas hipóteses, a primeira admitindo que a água formada não tenha afinidade com a superfície do catalisador, mantendo-se na fase gasosa, e a segunda considerando a adsorção desta nos sítios da zeólita Beta, R4. Para a formulação dos modelos considerou-se que há apenas um tipo de sítio ácido na zeólita Beta e que os compostos adsorvem de maneira competitiva.

$$
\begin{gathered}
\text { Isop }+* \rightleftharpoons \text { Isop* } \\
\text { Isop } * \text { Prope } *+\mathrm{H}_{2} \mathrm{O} \\
\text { 2Isop } \text { Ister }^{*}+\mathrm{H}_{2} \mathrm{O}+* \\
\text { Prope }{ }^{*} \rightleftharpoons \text { Prope }+* \\
\text { Eter } \rightleftharpoons \text { Eter }+* \\
\mathrm{H}_{2} \mathrm{O}+* \rightleftharpoons \mathrm{H}_{2} \mathrm{O}^{*}
\end{gathered}
$$

\begin{tabular}{|c|c|c|}
\hline Modelo & Hipótese & Velocidade de reação $\left(r_{\text {prope }}\right)$ e $\left(r_{\text {eter }}\right)$ \\
\hline D1 & $\begin{array}{l}\text { Pseudo- } \\
\text { homogêneo }\end{array}$ & $r_{\text {prope }}=k_{\text {prope }}\left(P_{\text {isop }}-\frac{P_{\text {prope }} P_{H_{2} O}}{K_{\text {eq, prope }}}\right) ; \quad r_{\text {eler }}=k_{\text {eler }}\left(P_{\text {isop }}^{2}-\frac{P_{\text {eter }} P_{H_{2} O}}{K_{\text {eq,eler }}}\right)$ \\
\hline D2 & $\begin{array}{l}\text { Não há } \\
\text { adsorção } \\
\text { da água }\end{array}$ & $r_{\text {prope }}=\frac{k_{2.1} K_{1}\left(P_{\text {isop }}-\frac{P_{\text {prope }} P_{H_{2} O}}{K_{\text {eq, prope }}}\right)}{\left(1+K_{1} P_{\text {isop }}+K_{3.1} P_{\text {prope }}+K_{3,2} P_{\text {eler }}\right)} ; \quad r_{\text {eler }}=\frac{k_{2.2} K_{1}\left(K_{1} P_{\text {isop }}^{2}-\frac{P_{\text {eter }} P_{H_{2} O}}{K_{\text {eq,eler }}}\right)}{\left(1+K_{1} P_{\text {isop }}+K_{3,1} P_{\text {prope }}+K_{3.2} P_{\text {eter }}\right)^{2}}$ \\
\hline D3 & $\begin{array}{l}\text { Há } \\
\text { adsorção } \\
\text { da água }\end{array}$ & $r_{\text {prope }}=\frac{k_{2.1} K_{1}\left(P_{\text {isop }}-\frac{P_{\text {prope }} P_{H_{2} O}}{K_{\text {eq, prope }}}\right)}{\left(1+K_{1} P_{\text {isop }}+K_{3.1} P_{\text {prope }}+K_{3.2} P_{\text {eler }}+K_{4} P_{H_{2}}\right)} ; \quad r_{\text {eler }}=\frac{k_{2.2} K_{1}\left(K_{1} P_{\text {isop }}^{2}-\frac{P_{\text {eter }} P_{H_{2} O}}{K_{\text {eq,eler }}}\right)}{\left(1+K_{1} P_{\text {isop }}+K_{3.1} P_{\text {prope }}+K_{3.2} P_{\text {eler }}+K_{4} P_{H_{2} O}\right)^{2}}$ \\
\hline
\end{tabular}

Tabela 2 - Modelos cinéticos para reação de desidratação de isopropanol.

A partir destas etapas de reação, foram formulados os modelos cinéticos para a velocidade de desidratação de isopropanol. Nos dois modelos de cinética heterogênea assumiu-se que as etapas determinantes da velocidade de reação eram as reações de superfície formando os 


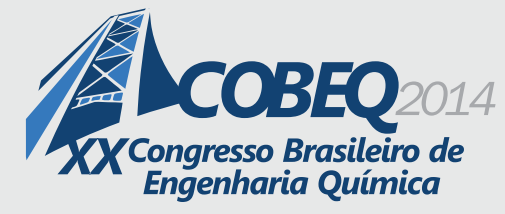

produtos. Contudo, os modelos diferem entre si por considerar ou não a etapa de adsorção de água nos sítios ativos do catalisador. Os modelos formulados para as velocidades de reação encontram-se na Tabela 2.

\subsection{Estimação de parâmetros}

O sistema reacional foi representado por modelos típicos de reator tubular com fluxo empistonado. O modelo do reator composto por um sistema de equações diferenciais foi resolvido numericamente com o auxílio da rotina DASSL (Petzold, 1989). Os modelos cinéticos propostos foram ajustados aos dados experimentais das frações molares de isopropanol, propeno e éter di-isopropílico na fração orgânica na saída do reator. E os parâmetros estimados foram as constantes cinéticas $\left(\ln \left(k_{m}{ }^{\text {Tref }}\right)\right.$ e $\left.E_{m} /\left(R . T_{\text {ref }}\right)\right)$ na forma da equação de Arrhenius reparametrizada, Equação 1 (Schwaab e Pinto, 2007), e as constantes de equilíbrio das etapas intermediárias $\left(\ln \left(\mathrm{K}_{\mathrm{n}}^{\text {Tref }}\right)\right.$ e $\left.\Delta \mathrm{H}_{\mathrm{n}} /\left(\mathrm{R} \cdot \mathrm{T}_{\mathrm{ref}}\right)\right)$. As constantes de equilíbrio são funções da temperatura, assim como as constantes cinéticas, e variam conforme a equação de van't Hoff reparametrizada, Equação 2. A temperatura de referência foi de $160{ }^{\circ} \mathrm{C}$. Na estimação de parâmetros os valores das constantes de equilíbrio das reações globais de formação de propeno e éter foram calculados a partir de dados termodinâmicos. A estimação foi realizada através da minimização da função objetivo de mínimos quadrados ponderados, com o uso de um algoritmo híbrido de otimização composto por um método estocástico acoplado a um método determinístico, em que a melhor solução encontrada pelo método do Enxame de Partículas (Kennedy e Eberhart, 1995) era utilizada como estimativa inicial para um método do tipo Gauss-Newton.

$$
\begin{aligned}
& k_{m}=\exp \left[\ln \left(k_{m}^{T_{r f f}}\right)+\frac{E_{m}}{R T_{r e f}}\left(\frac{T-T_{r e f}}{T}\right)\right] \\
& K_{n}=\exp \left[\ln \left(K_{n}^{T_{r e f}}\right)+\frac{\Delta H_{n}}{R T_{r e f}}\left(\frac{T-T_{r e f}}{T}\right)\right]
\end{aligned}
$$

Após a escolha do melhor modelo cinético, foi analisado o efeito das variáveis operacionais foi analisado através deste modelo cinético selecionado. Para tanto, foram escolhidas as variáveis operacionais a fração molar de isopropanol na alimentação e a velocidade mássica espacial por hora. A vazão molar de isopropanol na alimentação foi mantida fixa e as outras variáveis foram calculadas a partir das variáveis operacionais. 


\section{RESULTADOS E DISCUSSÃO}

\subsection{Caracterização da zeólita Beta}

A caracterização do catalisador por difração de raios-X indicou a formação de zeólita Beta (Yin et al., 2013). A zeólita Beta também foi caracterizada com relação às suas propriedades texturais por adsorção/dessorção de nitrogênio a $77 \mathrm{~K}$, apresentando área específica de $395 \mathrm{~m} / \mathrm{g}$, volume total de poros de $0,246 \mathrm{~cm}^{3} / \mathrm{g}$ e raio médio de poros de $36 \AA$.

\subsection{Reação de desidratação de isopropanol}

A forma ácida da zeólita Beta foi utilizada como catalisador, sendo empregados $10 \mathrm{mg} \mathrm{em}$ cada teste catalítico. Os experimentos foram efetuados de maneira independente e as variáveis operacionais foram ajustadas de acordo com o planejamento experimental. Nos experimentos E1 e E2, realizados com a menor temperatura do saturador, a alimentação continha menor fração molar de isopropanol; em contrapartida, na alimentação dos experimentos E3 e E4, maior temperatura do saturador, usou-se a maior fração molar de isopropanol.

A conversão de isopropanol nas diferentes temperaturas de reação em cada uma das sete condições experimentais é apresentada na Figura 1. A condição experimental que possibilitou a maior conversão em menores temperaturas foi àquela correspondente ao experimento E1, como esperado, já que este experimento foi realizado com a menor vazão total na alimentação, isto é maior tempo de contato entre mistura reacional e leito catalítico. O experimento que resultou nas menores conversões em todas as temperaturas inferiores a $200{ }^{\circ} \mathrm{C}$ foi o E4, pois neste experimento foram empregadas na alimentação maior fração de álcool e maior vazão. De maneira geral, observa-se o aumento da conversão com o aumento da temperatura. Todos os experimentos atingiram conversões próximas a $100 \%$ em temperaturas mais elevadas.

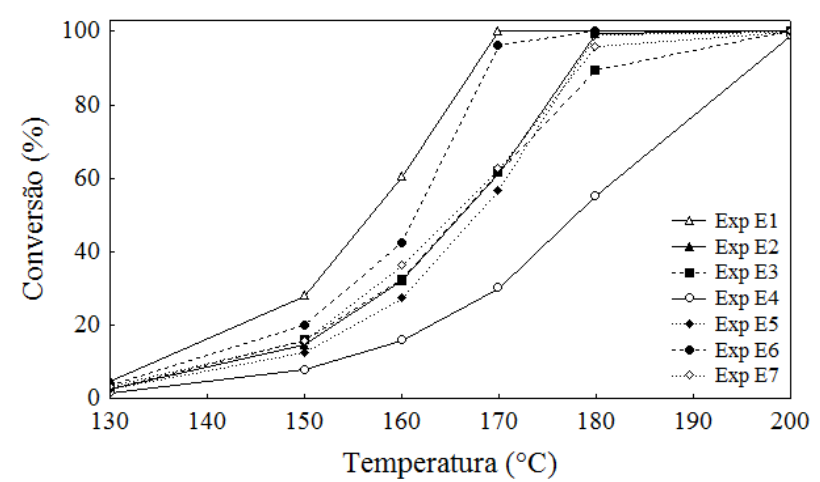

Figura 1 - Conversão de isopropanol em diferentes temperaturas os experimentos E1-E7.

Como não foi observada a formação de acetona em nenhum dos experimentos de desidratação, pode-se considerar que este catalisador apresenta apenas sítios ácidos. Propeno é o 


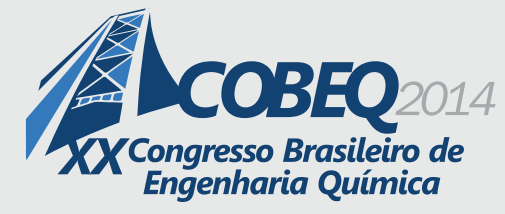

produto preferencial, principalmente em temperaturas elevadas. Além disso, a razão entre os produtos de desidratação, propeno e éter di-isopropílico, que foi superior a 0,8 para todas as condições experimentais, indica que a zeólita Beta possui acidez de moderada a alta.

\subsection{Modelagem cinética}

As variáveis dependentes dos modelos cinéticos foram os valores de fração molar de isopropanol, propeno e éter di-isopropílico. Para o melhor ajuste dos dados experimentais foram formulados, além do modelo pseudo-homogêneo (D1), dois modelos baseados nos mecanismos de Langmuir-Hinshelwood para cinética heterogênea. Observou-se que os modelos D2 e D3 proporcionaram ajustes significativamente melhores que o modelo D1, sendo este considerado insuficiente para a predição das frações molares dos compostos envolvidos na reação de desidratação de isopropanol.

Foram testados os diferentes modelos cinéticos, variando-se também a quantidade de parâmetros em cada modelo, sendo a influência destes parâmetros observada no valor da função objetivo e na confiabilidade dos demais parâmetros estimados. Partindo-se do modelo mais simples, o pseudo-homogêneo (D1), foi possível observar o efeito de cada constante de equilíbrio de adsorção no ajuste dos modelos. O modelo mais simples (DS), Equações 3 e 4, estatisticamente igual ao modelo D2, inclui apenas a constante de equilíbrio da adsorção do isopropanol, além das constantes cinéticas, totalizando 6 parâmetros a serem estimados. Comparando o modelo simplificado e o completo, é possível afirmar que os termos relativos à adsorção de propeno e de éter das equações de velocidade de reação não contribuem para a melhora do ajuste, podendo ser descartados do modelo. Da mesma forma, no que se refere à predição da fração molar de propeno na saída do reator, a adição do termo relativo à adsorção da molécula de água nas equações de velocidade de reação, modelo D3, não melhorou a qualidade dos ajustes. Assim, é possível considerar que a inclusão da etapa de adsorção de água nos sítios ativos do catalisador é desnecessária na modelagem cinética da desidratação de isopropanol.

$$
\begin{array}{r}
r_{\text {prope }}=\frac{k_{2.1} K_{1}\left(P_{\text {isop }}-\frac{P_{\text {prope }} P_{H_{2} O}}{K_{\text {eq, prope }}}\right)}{\left(1+K_{1} P_{\text {isop }}\right)} \\
r_{\text {eler }}=\frac{k_{2.2} K_{1}\left(K_{2.1} P_{\text {isop }}^{2}-\frac{P_{\text {eler }} P_{H_{2} O}}{K_{\text {eq,eer }}}\right)}{\left(1+K_{1} P_{\text {isop }}\right)^{2}}
\end{array}
$$

Nenhum dos modelos cinéticos propostos possibilitou boa predição dos valores de fração molar de éter di-isopropílico, devido ao erro experimental relacionada à baixa concentração deste composto na mistura de no máximo $2,5 \%$. No entanto, como o propeno é o produto de interesse desta reação considerou-se que o modelo mais simples, DS, é suficiente para representar adequadamente a reação de desidratação de isopropanol. Os valores obtidos para os parâmetros desse modelo e seus respectivos desvios padrão estão relacionados na Tabela 3 e o ajuste aos 
dados experimentais pode ser observado na Figura 2.

Tabela 3 - Parâmetros cinéticos e desvio padrão de parâmetros do modelo DS

\begin{tabular}{ccc|ccc}
\hline Parâmetro & Valor & Desvio Padrão & Parâmetro & Valor & Desvio Padrão \\
\hline $\ln \left(K_{1}^{T_{\text {rff }}}\right)$ & 5,98 & 0,82 & $\Delta H_{1} / R T_{r e f}$ & 49,37 & 16,79 \\
$\ln \left(k_{2.1}^{T_{\text {rf }}}\right)$ & $-1,92$ & 0,10 & $E_{2.1} / R T_{r f f}$ & 27,41 & 2,58 \\
$\ln \left(k_{2.2}^{T_{r f}}\right)$ & $-5,26$ & 0,17 & $E_{2.2} / R T_{r e f}$ & 3,81 & 21,41 \\
\hline
\end{tabular}
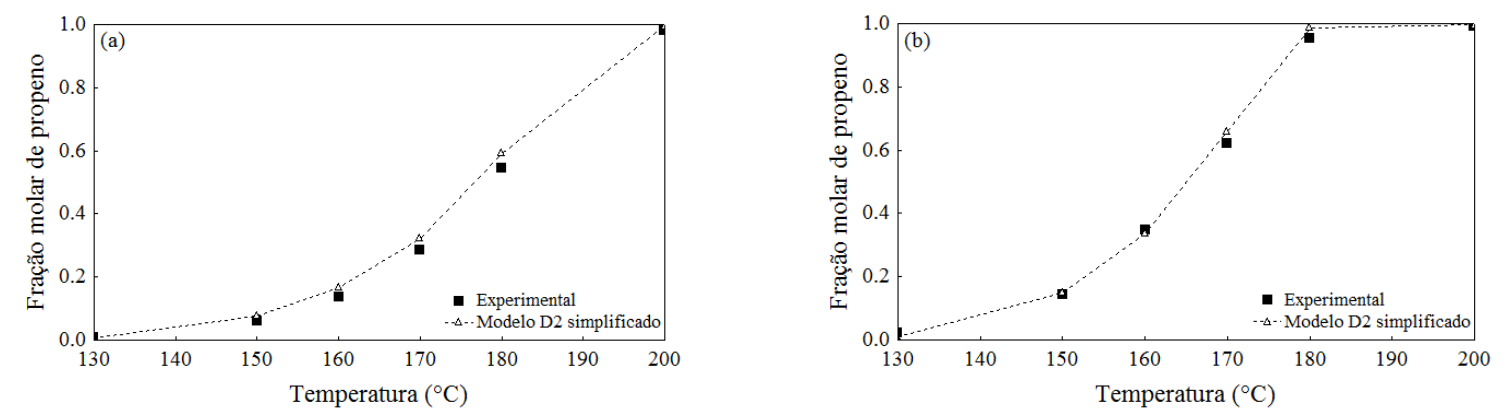

Figura 2 - Ajuste do modelo DS para as condições experimentais (a) E4 e (b) E7.

A partir do modelo DS, mais adequado, e dos parâmetros estimados foi possível analisar melhor a influência das variáveis do processo sobre a composição dos produtos de reação. Os efeitos da fração molar de alimentação de isopropanol e da velocidade espacial mássica foram coerentes com o esperado, há aumento da fração molar de propeno com a diminuição da velocidade mássica espacial, devido ao um maior tempo de contato entre a mistura reacional e o leito catalítico, e com a diminuição da fração molar de isopropanol na alimentação, pois há uma maior quantidade de sítios ativos disponíveis.

\section{CONCLUSÃO}

Nos testes reacionais, empregando zeólita Beta como catalisador para a desidratação de isopropanol, observou-se a formação de propeno como produto principal, com formação de éter di-isopropílico apenas em pequenas quantidades. Foram propostos modelos cinéticos para a desidratação de isopropanol, sendo possível determinar que a hipótese de que há a adsorção de moléculas de água nos sítios ativos do catalisador não é necessária para o bom ajuste dos dados experimentais. Obteve-se, assim, um modelo cinético simplificado, cujos parâmetros foram estimados, que proporciona uma boa predição das frações molares do propeno na fração orgânica na saída do reator.

\section{AGRADECIMENTOS}

Os autores agradecem à Fundação de Amparo à Pesquisa do Estado do Rio Grande do Sul (FAPERGS) e a Secretaria de Ciência Tecnologia e Inovação do RS (SCIT-RS). 


\section{NOMENCLATURA}

composto*
$E_{m} / R T_{r e f}$
$\Delta H_{n} / R T_{\text {ref }}$
$K_{\text {eq,composto }}$
$k_{m}$
$K_{n}$
$\ln \left(k_{m}^{T_{\text {ref }}}\right)$
$\ln \left(K_{n}^{T_{r e f}}\right)$
$P_{j}$

Sítio ativo livre

Espécies adsorvidas

Parâmetro cinético, razão entre a energia de ativação da reação $\left(E_{m}\right)$ e o produto entre a constante dos gases $(R)$ e a temperatura de referência $\left(T_{r e f}\right)$

Parâmetro de equilíbrio, razão entre a variação da entalpia da reação $\left(\Delta H_{n}\right)$ e o produto entre a constante dos gases $(R)$ e a temperatura de referência $\left(T_{r e f}\right)$

Constante de equilíbrio da reação global

Constante cinética da reação $m$, sendo $m$ a etapa limitante

Constante de equilíbrio da etapa $n$

\section{REFERÊNCIAS}

BEDIA, J.; RUIZ-ROSAS, R.; RODRÍGUEZ-MIRASOL, J.; CORDERO, T. A kinetic study of 2-propanol dehydration on carbon acid catalysts. J Catal, v. 271, p. 33-42, 2010.

ENZWEILER, H.; COUTINHO, E. B.; SCHWAAB, M. Cinzas de casca de arroz como fonte alternativa de silício para a síntese de zeólita beta. REGET, v. 17, p. 3284-3292, 2013.

FIGUEIREDO, J. L.; RIBEIRO, F. R. Catálise heterogênea. Fundação Calouste Gulbenkian, Lisboa, 1989.

KENNEDY, J.; EBERHART, R. Particle swarm optimization. In: Proceedings of the IEEE International Conference on Neural Networks, Perth, Australia, v. 4, p. 1942-1948, 1995.

PETZOLD, L. R. DASSL Code (Differential Algebraic System Solver), Computing and Mathematics Research Division, Lawrence Livermore National Laboratory, Livermore, CA, USA, 1989.

SCHWAAB, M.; PINTO, J. C. Optimum reference temperature for reparameterization of the Arrhenius equation. Part 1: Problems involving one kinetic constant. Chem Eng Sci, v. 62, p. 2750-2764, 2007.

TREJO, F.; RANA, M. S.; ANCHEYTA, J.; RUEDA, A. Hydrotreating catalysts on different supports and its acid-base properties. Fuel, v. 100, p. 163-172, 2012. 